\section{Consideraciones en torno a las Guías USA 2017 de hipertensión arterial}

\author{
EDUARDO FASCE ${ }^{1}$, LUIS HERNÁN ZÁRATE², LILIANA ORTIZ1
}

The 2017 Guidelines on hypertension of the American College of Cardiology and American Heart Association, which proposed values of 130/80 $\mathrm{mmHg}$ as the cutoff points for the onset of hypertension, aroused great interest. This recommendation is based in the SPRINT study (The Systolic Pressure Intervention Trial), which included hypertensive patients over 50 years of age, non-diabetic, without a history of stroke and with a low representation of subjects with a history of coronary artery disease (16\%). A group with intensive anti-hypertensive therapy (pressure achieved $121.5 \mathrm{mmHg}$ ) achieved a significantly lower cardiovascular risk as compared with a group with standard therapy (pressure achieved 134.6 $\mathrm{mm} \mathrm{Hg}$ ). The Guide proposes immediate pharmacological therapy in diabetic hypertensive patients, in those with stage 3 chronic kidney disease or with persistent albuminuria, and in patients with atherosclerotic disease. The Guideline does not include the management of isolated systolic hypertension of the elderly and did not consider studies that show an increased risk when pressure is reduced below 130/80 $\mathrm{mmHg}$ in patients with coronary disease, peripheral vascular disease, diabetes mellitus or chronic renal failure. The new classification of hypertension would increase the number of hypertensive patients in our country by more than one million, would increase the risk associated with diastolic pressure reductions in older adults and ignores the evidence indicating a risk associated with reductions below $130 / 80 \mathrm{mmHg}$ in patients with diabetes, with chronic renal failure or with atherosclerotic disease. Therefore, it is advisable to maintain a threshold of $140 / 90 \mathrm{mmHg}$ and perform a careful and gradual management of blood pressure in the latter group of hypertensive patients.

(Rev Med Chile 2018; 146: 1317-1324)

Key words: Guideline; Hypertension; Practice Guideline.
'Departamento de Educación Médica, Facultad de Medicina, Universidad de Concepción, Chile. Concepción, Chile. ${ }^{2}$ Departamento de Medicina Interna, Facultad de Medicina, Universidad de Chile. Santiago, Chile.

Los autores declaran no tener conflictos de interés.

Trabajo no recibió

financiamiento.

Recibido el 11 de junio de 2018, aceptado el 12 de septiembre de 2018.

Correspondencia a:

Eduardo Fasce $\mathrm{H}$.

Departamento de Educación Médica, Facultad de Medicina, Universidad de Concepción. Barrio Universitario. Janequeo esquina Chacabuco s/n Concepción. VIII Región, Chile. efasce@udec.cl
L as nuevas guías de hipertensión arterial de la Asociación Americana de Cardiología, el Colegio Americano de Cardiólogos y nueve sociedades adicionales, han causado desconcierto y controversia debido a la nueva clasificación de hipertensión propuesta y, en especial, al rebajar el límite de hipertensión a $130 / 80 \mathrm{mmHg}$ independientemente de la edad ${ }^{1}$. Se recuerda que tan sólo tres años antes, el $8^{\circ}$ informe del Joint National Committee recomendaba elevar a $150 \mathrm{mmHg}$ el límite para la presión sistólica en mayores de 60 años ${ }^{2}$, aun cuando un grupo disidente publicó su opinión contraria ${ }^{3}$.

Los fundamentos para respaldar el nuevo límite para la hipertensión son sustentados en estudios realizados en población general los que demuestran que el riesgo cardiovascular aumenta de manera lineal con los niveles de presión arterial a partir de presiones inferiores a 130/80 $\mathrm{mmHg}$. Estas evidencias emergen de numerosos trabajos destacando algunos con más de un millón de personas $^{4,5}$. 
Un segundo apoyo se sustenta en el estudio SPRINT (The Systolic Pressure Intervention Trial), publicado en noviembre de $2015^{6}$. Este incluyó 9.361 sujetos mayores de 50 años, no diabéticos, sin accidente cerebrovascular previo, con riesgo cardiovascular a 10 años sobre $15 \%$, enfermedad cardiovascular clínica o subclínica, e insuficiencia renal. Se randomizó a terapia intensiva, para presión sistólica (PS) objetivo $<120 \mathrm{mmHg}$, y terapia estándar para PS < $140 \mathrm{mmHg}$. Al cabo de 3,26 años los promedios de PS fueron de 121,5 y 134,6 $\mathrm{mmHg}$, respectivamente. El grupo de terapia intensiva tuvo una disminución de $25 \%$ del objetivo primario (compuesto por infarto del miocardio, síndromes coronarios agudos, accidente vascular cerebral, insuficiencia cardíaca y mortalidad cardiovascular), $43 \%$ de disminución de muertes de origen cardiovascular, $27 \%$ reducción de mortalidad total y $38 \%$ reducción de hospitalizaciones por insuficiencia cardíaca.

Una nueva comunicación derivada del estudio SPRINT en una subpoblación de 2.636 mayores de 75 años, obtuvo similares resultados ${ }^{7}$.

En consideración al alto porcentaje de pacientes con reacciones adversas severas en el estudio SPRINT, Philips y cols. ${ }^{8}$ compararon la relación entre beneficio y daño generado. Las reacciones adversas severas fueron definidas como aquellas que "pueden ser fatales, comprometer la vida, provocar discapacidad significativa o persistente, requerir o prolongar hospitalización o requerir intervención médica o quirúrgica"'. Los autores separaron el riesgo cardiovascular a 10 años en cuartiles, estableciendo la relación entre cada cuartil y el nivel de daño correspondiente. Para los dos primeros cuartiles, con riesgo a 10 años menor a $18,1 \%$, se obtuvo un daño mayor que el beneficio. A partir del tercer cuartil (riesgo a 10 años mayor de 18,1\%), la relación se invierte. De acuerdo a esos resultados, los autores proponen niveles de presión sistólica menores de $140 \mathrm{mmHg}$ para riesgo a 10 años menor de $18,1 \%$ y presión sistólica menor de $130 \mathrm{mmHg}$ para riesgo a 10 años mayor de $18,1 \%$.

\section{Efecto de la aplicación de la guía sobre la salud pública}

Con la intención de cuantificar el eventual efecto de la nueva clasificación de hipertensión en nuestro país, tomamos como base la información disponible del estudio epidemiológico de hipertensión realizado en la comunidad urbana de la VIII Región en $2004^{9}$, tanto por disponer de la base de datos como por tratarse de tasas de prevalencia de hipertensión (por haber sido obtenidas en registros efectuados en días separados) y no de elevación casual obtenida en una visita.

La inclusión de sujetos con presiones entre 130-139/80-89 mmHg, determina un aumento de la prevalencia desde $21,7 \%$ (para umbral de $140 / 90 \mathrm{mmHg}$ ) a $31,3 \%$.

Aun considerando que las tasas de prevalencia actuales de hipertensión pudiesen ser diferentes a las de 2004, y tan sólo con la intención de realizar una aproximación al posible impacto nacional de la nueva clasificación, al tomar la información del Censo poblacional de 2017 se obtendría un aumento de 1.156.824 nuevos hipertensos, sujetos que hasta este momento son considerados saludables.

Sin dudas que un aumento de alrededor de 1 millón de nuevos hipertensos debiese generar un fuerte impacto en la salud pública tanto en términos de una mayor demanda en los servicios de atención como en los costos asociados al estudio y tratamiento.

En consideración a ello surge el interés de revisar con mayor precisión la pertinencia de aplicar la nueva clasificación propuesta en la Guía.

De acuerdo a ella, el requerimiento de terapia farmacológica para este nuevo grupo de hipertensos se debiese iniciar en pacientes diabéticos, con enfermedad cardiovascular aterosclerótica (enfermedad coronaria, accidente vascular cerebral isquémico, enfermedad vascular periférica, aterosclerosis carotídea), en enfermedad renal crónica etapa $\geq 3$ o con albuminuria persistente, $o$ cuando el riesgo cardiovascular a 10 años es mayor a $10 \%$. Con riesgo cardiovascular menor a $10 \%$ procedería aplicar medidas no farmacológicas por seis meses e iniciar terapia farmacológica al no haber adecuado control.

\section{Omisiones y discrepancias}

\section{Hipertensión en adultos mayores}

No se establecen diferencias entre adultos mayores e hipertensos jóvenes. De hecho, no se incluye el manejo de la hipertensión sistólica aislada del adulto mayor la cual se caracteriza por importantes elevaciones de la presión de pulso. En estos casos, el esfuerzo terapéutico para alcanzar 
metas de presión sistólica inferior a $130 \mathrm{mmHg}$ puede determinar significativos descensos de la presión diastólica y comprometer la perfusión coronaria:

- En el estudio SHEP (Systolic Hypertension in the Elderly) se demostró una correlación significativa entre descenso de PD y aumento de los eventos cardiovasculares, incluido accidente vascular cerebral y enfermedad coronaria ${ }^{10}$.

- El estudio europeo de hipertensión sistólica (Syst-Eur) demostró aumento de la mortalidad con PD inferior a $55 \mathrm{mmHg}$ y aumento de los eventos cardiovasculares a partir de PD menor de $70 \mathrm{mmHg}$ en la subpoblación coronaria ${ }^{11}$.

- En el estudio HYVET (Hypertension in the very elderly), que incluyó 3.845 pacientes mayores de 80 años sin otras condiciones mórbidas, los beneficios logrados con la terapia activa en comparación al placebo se lograron con promedios de presión de 143/78 $\mathrm{mmHg}^{12}$.

- Un subestudio de INVEST (International Verapamil-Trandolapil study), referido a hipertensos mayores de 80 años con enfermedad coronaria, demostró un aumento de los eventos cardiovasculares con presiones bajo 140/70 $\mathrm{mmHg}^{13}$.

Se puede concluir que en los adultos mayores hipertensos una terapia agresiva puede determinar aumento de las complicaciones cardiovasculares al determinar una curva J.

\section{Presión de pulso y riesgo cardiovascular}

La hipertensión sistólica aislada del adulto mayor es consecuencia de la rigidez de la pared aórtica la cual determina un aumento de la presión de pulso y de la velocidad de la onda de pulso siendo ambas predictoras de eventos cardiovasculares ${ }^{14}$ :

- Vaccarino y cols. comprueban un aumento significativo de eventos coronarios, insuficiencia cardíaca y mortalidad en función del aumento de la presión de pulso (especialmente sobre 70 $\mathrm{mmHg}$ ) en 2.812 mayores de 65 años con 10 años de seguimiento ${ }^{15}$.

- Un estudio realizado en Francia con más de 72 mil sujetos sin patología cardiovascular, demostró correlaciones estadísticamente significativas entre presión de pulso (PP) y riesgo cardiovascular ${ }^{16}$, resultados similares a los encontrados por Glynn R y cols. en 9.431 adultos mayores ${ }^{17}$.
- En el estudio Framingham, con una población de 3.628 hombres y 4.170 mujeres sin enfermedad cardiovascular, en un seguimiento de 12 años, para una PS $>140 \mathrm{mmHg}$ el riesgo cardiovascular aumenta al disminuir la PD, siendo $19,4 \%$ para PD entre 80 y $89 \mathrm{mmHg}$ y $31,1 \%$ para $\mathrm{PD}<80 \mathrm{mmHg}$. Con PS $>160$ mmHg y $\mathrm{PD}<80 \mathrm{mmHg}$ (esto es, presión de pulso $\geq 80 \mathrm{mmHg}$ ) el riesgo aumenta a $36 \%{ }^{18}$. Estos efectos fueron reafirmados en un análisis posterior del Framingham ${ }^{19}$.

- Una publicación más reciente del estudio SPRINT $^{20}$ demuestra un aumento de la mortalidad total, del objetivo primario y del compromiso de la función renal en el grupo de terapia intensiva para presiones diastólicas inferiores a $70 \mathrm{mmHg}$ aun cuando el beneficio del descenso intensivo de la presión sistólica no es anulado por la PD basal.

\section{Hipertensos con enfermedad coronaria}

Siendo el consumo de oxígeno miocárdico dependiente del flujo y, por tanto, de la vasodilatación coronaria, las lesiones coronarias obstructivas limitan la respuesta adaptativa a los descensos de presión diastólica.

No debe sorprender entonces que la terapia antihipertensiva en pacientes coronarios haya demostrado aumento del riesgo cardiovascular dependiente de los niveles de descenso de la PD:

- El estudio INVEST incluyó 22.576 pacientes con insuficiencia coronaria estable, demostrando un aumento de la mortalidad total y los infartos al miocardio con presiones menores de $119 / 84 \mathrm{mmHg}^{21}$. Como ya fuera mencionado, para el subgrupo de pacientes mayores de 80 años ${ }^{13}$, el aumento del riesgo se obtuvo con presiones inferiores a $140 / 70 \mathrm{mmHg}$.

- En 6.166 pacientes revascularizados del estudio INVEST, (2.784 bypass aortocoronario, 2.594 angioplastía percutánea y 788 con ambos), los niveles de presión que inician un aumento del riesgo correspondieron a 145/80 $\mathrm{mmHg}$ para angioplastía, $140 / 70 \mathrm{mmHg}$ para ambas, $125 / 55$ para bypass y $125 / 75$ para coronarios no revascularizados ${ }^{22}$. Los autores concluyen que en esta población de pacientes no hay soporte para presiones objetivo bajo $130 / 80 \mathrm{mmHg}$.

- El estudio TNT (Treatment to New Targets) comparó terapia intensiva con terapia estándar 
en 10 mil hipertensos con enfermedad coronaria, demostrando una curva $\mathrm{J}$ a niveles de $146,3 / 81,4 \mathrm{mmHg}^{23}$.

- En una subpoblación de 3 mil pacientes coronarios del estudio HOT (Hypertension Optimal Treatment), se comprobó curva J para presión diastólica menor de $81 \mathrm{mmHg}^{24}$.

- En registros simultáneos de monitoreo ambulatorio de presión arterial y electrocardiograma, se demostró cambios isquémicos transitorios de la onda $\mathrm{T}$ dependientes de descensos de $\mathrm{PD}^{25}$.

- En un seguimiento de 5 años en 22.672 coronarios estables se comprobó curva "J" para mortalidad cardiovascular, infarto del miocardio a partir de $120 / 70 \mathrm{mmHg}^{26}$.

\section{Terapia antihipertensiva en pacientes diabéticos}

La coexistencia de hipertensión y diabetes mellitus es considerada en la Guía como de alto riesgo cardiovascular, propiciando terapia antihipertensiva inmediata a partir de $130 / 80 \mathrm{mmHg}$, es decir, manteniendo el criterio del Joint National Committee del año 2003.

El principal fundamento provino de los resultados de los estudios HOT ${ }^{24}$, UKPDS (United Kingdom Prospective Diabetes Study) ${ }^{27}$ y ADVANCE (Action in Diabetes and Vascular Disease Controlled Evaluation) ${ }^{28}$. En ellos la terapia más intensiva lograba reducir significativamente las complicaciones macro y microvasculares en relación a la terapia estándar. Sin embargo, los promedios de presión obtenidos con la terapia intensiva resultaron mayores que $130 / 80 \mathrm{mmHg}$, restando fundamento al límite de 130/80 $\mathrm{mmHg}$.

Por otra parte, otras publicaciones no demostraron ventajas con presiones inferiores a 130/80 $\mathrm{mmHg}$ e incluso en algunas se comprobó aumento del riesgo con presiones bajo ese nivel:

- En el estudio ACCORD (Action to Control Cardiovascular Risk in Diabetes), 4.733 hipertensos con terapia intensiva (presión lograda 119/64 mmHg) o estándar (presión lograda $134 / 71 \mathrm{mmHg}$ ) no se constató diferencias en los eventos cardiovasculares mayores ${ }^{29}$. Una prolongación de este estudio a 60 meses, presentado en el congreso de la Sociedad Americana de Cardiología en Orlando el año 2015, no demostró diferencias con el estudio inicial $^{30}$.
- En el estudio ONTARGET (Ongoing Telmisartan Alone and in Combination with Ramipril Global Endpoint Trial), no hubo beneficios con PS $<130 \mathrm{mmHg}$ pero sí aumento de la mortalidad con PS $<125 \mathrm{mmHg}^{31}$.

- La población diabética del estudio INVEST $(\mathrm{n}=6.600)$ redujo el riesgo cardiovascular hasta niveles de PS entre 115 y $120 \mathrm{mmHg}$, observando un aumento del riesgo bajo 115 $\mathrm{mmHg}^{32}$.

- En el estudio ABCD (Appropiate BP Control in Diabetes) no hubo diferencias significativas en los eventos cardiovasculares entre terapia intensiva $(128 / 75 \mathrm{mmHg})$ y placebo $(137 / 81$ $\mathrm{mmHg})^{33}$.

- En el último consenso de la Sociedad Americana de Diabetes ${ }^{34}$ se estableció un umbral de 140/90 mmHg, reservando valores bajo 130/80 sólo a condiciones especiales de alto riesgo siempre que no se generen reacciones adversas, sin embargo, los autores no especificantales condiciones especiales.

\section{Insuficiencia renal crónica}

El último consenso data de $2012^{35}$ y considera niveles de $140 / 90 \mathrm{mmHg}$ en presencia de albuminuria $<30 \mathrm{mg}$ en $24 \mathrm{~h}$ y de $130 / 80$ para albuminurias mayores. Para los adultos mayores se recomienda tomar en consideración comorbilidades y realizar reducciones graduales cautelando la aparición de eventos adversos como alteraciones electrolíticas, deterioro agudo de la función renal, hipotensión ortostática y reacciones adversas a los medicamentos. El nivel de evidencia correspondió a opinión de expertos.

La National Kidney Foundation-Kidney Disease Outcomes Quality Initiative, a través de una revisión sistemática de la literatura, estimó que la evidencia disponible no era concluyente y no probaba que una presión arterial $<130 / 80 \mathrm{~mm}$ fuera superior a $<140 / 90 \mathrm{mmHg}$ en la prevención del riesgo ${ }^{36}$.

En un estudio con más de 650.000 adultos mayores con falla renal crónica, los extremos más altos y más bajos de presión se asociaban con aumento de la mortalidad, siendo la presión de pulso el mayor predictor de riesgo. Los autores concluyen que no es ventajoso alcanzar niveles de presión sistólica inferiores a $130 \mathrm{mmHg}$ cuando los niveles de PD son bajos ${ }^{37}$. En apoyo de ello, otro estudio demostró que el aumento de la PP era 
un significativo predictor de riesgo cardiovascular en 1.099 pacientes con enfermedad renal crónica avanzada (filtración glomerular $<30 \mathrm{ml} / \mathrm{min} \mathrm{x}$ $\left.1,73 \mathrm{~m}^{2}\right)^{38}$.

En el estudio IDNT (Irbesartan in Diabetic Nephropaty Trial) se evaluó en 1.590 hipertensos con nefropatía diabética (cretininemia $>3 \mathrm{mg} \%$ ) la progresión de la falla renal y la mortalidad, demostrando el inicio de curva J para mortalidad cardiovascular bajo $120 \mathrm{mmHg}$, y un aumento de mortalidad e infartos al miocardio con presiones diastólicas bajo un rango entre 80 y $85 \mathrm{mmHg}^{39}$.

Upadhyay y cols. analizan tres estudios en pacientes con filtración glomerular $<69 \mathrm{ml} / \mathrm{min}$ $\mathrm{x} 1,73 \mathrm{~m}^{2}$ y excreción urinaria de albúmina $>30$ $\mathrm{mg} /$ día, concluyendo que no existen evidencias que demuestren ventajas para presiones objetivo $<130 / 80 \mathrm{mmHg}$ en relación a $<140 / 90 \mathrm{mmHg}^{40}$. En este análisis se incluyó a Modification of Diet in Renal Disease Study ${ }^{41}$, African American Study of Kidney Disease ${ }^{42}$ y Ramipril Efficacy in Nephropathy ${ }^{43}$.

En 2.160 pacientes con accidente vascular cerebral lacunar previo y función renal normal, la terapia antihipertensiva más intensiva (PA lograda $127 / 70 \mathrm{mmHg}$ ) en relación a terapia estándar (PA 137/76 mmHg), determinó una disminución significativa de la filtración glomerular durante el primer año, declinación que se prolongó a lo largo del seguimiento ${ }^{44}$.

Un subanálisis del estudio SPRINT ${ }^{44}$ referido a 2.646 pacientes con filtración glomerular de 47,9 $\mathrm{ml} / \mathrm{min} \times 1,73 \mathrm{~m}^{2}$, demostró que la disminución de $30 \%$ de la filtración glomerular fue significativamente mayor en el grupo de terapia intensiva ${ }^{45}$.

Malhotra y cols. realizan un metaanálisis de 18 estudios con 15.924 pacientes con enfermedad renal crónica etapas 3 a 5, demostrando que la terapia intensiva redujo en $14 \%$ la mortalidad en relación a la terapia estándar. Sin embargo, la presión alcanzada en el grupo de terapia intensiva fue de $132 \mathrm{mmHg}^{46}$.

\section{Enfermedad vascular periférica}

Un estudio adicional del INVEST, en 2.699 pacientes con enfermedad vascular periférica, el menor nivel de riesgo se obtuvo con presiones entre 135-145/60-90 $\mathrm{mmHg}$, comparativamente con presiones menores y mayores. Los autores declaran que sus resultados no avalan presiones objetivo bajo $130 / 80 \mathrm{mmHg}^{47}$. No obstante ello, la ausencia de un mayor número de estudios no permite establecer un límite más preciso, requiriendo un manejo individualizado.

\section{Accidente vascular cerebral}

En 20.332 pacientes con accidente vascular cerebral previo, la reducción de presión sistólica hasta $139 \mathrm{mmHg}$ disminuyó significativamente el riesgo de mortalidad cardiovascular. Reducciones a menos de $130 \mathrm{mmHg}$ y menos de $120 \mathrm{mmHg}$ aumentaron el riesgo en $16 \%$ y $31 \%$, respectivamente $^{48}$.

\section{Conclusiones}

1. Existen fundadas evidencias para considerar a presiones inferiores a 130/80 $\mathrm{mmHg}$ como niveles óptimos en la población general. Sin embargo, este comportamiento natural de la presión arterial no es necesariamente extrapolable a una condición no natural como es la hipertensión.

2. No parece prudente reducir a niveles inferiores de 130/80 mmHg a hipertensos diabéticos o que presenten patología aterosclerótica previa. En tales casos es preferible continuar manteniendo presiones de 140/90 $\mathrm{mmHg}$ como objetivo terapéutico evitando la generación de una curva "J".

3. Especial consideración se debe tener en los adultos mayores con presión sistólica aislada donde los niveles de presión diastólica deben limitar la intensidad del tratamiento con el propósito de evitar el fenómeno de la curva "J".

4. Por otra parte, la intensidad del descenso de presión debiese ser gradual y cauteloso con la finalidad de evitar la hipotensión ortostática la cual aumenta el riesgo de infarto del miocardio, accidente vascular cerebral y mortalidad, como fuera demostrado en 9.139 sujetos de edad media evaluados a través de 26 años $^{49}$.

Addendum: En el período posterior al envío de este artículo a la Revista Médica de Chile, en el mes de agosto de este año, la Sociedad Internacional de Hipertensión comunicó su decisión de mantener niveles de 140/90 mmHg como límite de inicio de la hipertensión arterial ${ }^{50}$. Una posición similar fue planteada en las Guías de la Sociedad Europea de 
Cardiología en conjunto con la Sociedad Europea de Hipertensión ${ }^{51}$. En esta última se establecen los umbrales para tratamiento considerando 140/90 mmHg para población entre 18 y 79 años y de 160/90 mmHg para mayores de 79 años. Estos criterios incluyen la asociación con diabetes mellitus, enfermedad renal crónica, enfermedad coronaria, accidente vascular cerebral e isquemia cerebral transitoria.

\section{Referencias}

1. ACC/AHA/AAPA/ABC/ACPM/AGS/APHA/ASH/ASPC/ NMA/PCNA guideline for the prevention, detection, evaluation, and management of high blood pressure in adults: a report of the American College of Cardiology/ American Heart Association Task Force on Clinical Practice Guidelines. J Am Coll Cardiol 2017, Nov 13.

2. James PA, Oparil S, Carter BL, Cushman WC, Dennison-Himmelfarb C, Handler J, et al. 2014 evidence-based guideline for the management of high blood pressure in adults; report from the panel members appointed to the Eighth Joint National Committee. JAMA 2014; 311: 507-20.

3. Wright JT, Fine L, Lackland D, Ogedegbe G, Dennison C. Evidence supporting a systolic blood pressure goal of less than $150 \mathrm{mmHg}$ in patients aged 60 years or older; the minority view. Ann Intern Med 2014; 160: 499-503.

4. Rapsomaniki E, Timmis A, George J, Pujades-Rodríguez M, Shah A, Denaxas D, et al. Blood pressure and incidence of twelve cardiovascular diseases: lifetime risks, healthy life-years lost, and age-specific associations in 1.25 million people. Lancet 2014; 383: 1899-911.

5. Guo X, Zhang X, Guo L, Li Z, Zheng L, Yu S, et al. Association Between Pre-hypertension and Cardiovascular Outcomes: A Systematic Review and Meta-analysis of Prospective Studies Curr Hyp Rep 2013; 15: 703-16.

6. Wright JT, Williamson JD, Whelton P, Snyder JK, Sink KM, Rocco MV, et al. SPRINT Research Group. A randomized trial of intensive vs standard blood pressure control. N Eng J Med 2015; 373: 2103-16.

7. Williamson JD, Supiano MA, Applegate WB, Berlowitz DR, Campbell RC, Chertow GM, et al. Intensive vs Standadrd Blood Pressure Control and Cardiovascular Disease Outcomes in Adults Aged $\geq 75$ Years. A Randomized Clinical Trial. JAMA 2016; 315: 2673-82.

8. Philips RA, Xu J, Peterson LE, Arnold RM, Diamond JA, Schussheim AE. Impact of Cardiovascular Risk on the Relative Benefit and Harm of Intensive Treatment of Hypertension. J Am Coll Cardiol 2018; 71: 1601-10.
9. Fasce E, Campos I, Ibáñez P, Zárate L, Román O, Fasce F. Trends in prevalence, awareness, treatment and control of hypertension in urban communities in Chile. J Hypertens 2007; 25: 1807-11.

10. Somes GW, Pahor M, Shorr R, Cushman W, Applegate $\mathrm{W}$. The role of diastolic blood pressure when treatment isolated systolic hypertension. Arch Intern Med 1999; 159: 2004-9.

11. Fagard RH, Staessen JA, Thijs L, Celis H, Bulpitt C, de Leeuw $\mathrm{P}$, et al. On treatment diastolic blood pressure and prognosis in systolic hypertension. Arch Intern Med 2007; 167: 1884-91.

12. Beckett N, Peters R, Fletcher A, Staessen J, Liu L, Dumitrascu D, et al. Treatment of hypertension in patients 80 years of age and older. N Eng J Med 2008; 358: 1887-98.

13. Denardo S, Gong Y, Nichols W, Messerli F, Bavry A, Cooper-Dehoff R, et al. Blood pressure and outcomes in very old hypertensives coronary artery disease patients: an INVEST substudy. Am J Med 2010; 123: 719-26.

14. Boutouyrie P, Tropeano A, Asmar R, Gautier R, Benethos A, Lacolley P, et al. Aortic stiffness is an independent predictor of primary coronary events in hypertensive patients: a longitudinal study. Hypertension 2002; 39: 10-5.

15. Vaccarino V, Holford T, Krumholz H. Pulse pressure and risk for myocardial infarction and heart failure in the elderly. J Am Coll Cardiol 2000; 36: 130-8.

16. Benetos A, Thomas F, Joly L, Blacher J, Pannier B, Labta $\mathrm{C}$, et al. Pulse pressure amplification: a mechanical biomarker of cardiovascular risk. J Am Coll Cardiol 2010; 55: 1032-7.

17. Glynn R, Chae C, Guralnik J, Taylor J, Hennekens C. Pulse pressure and mortality in older people. Arch Intern Med 2000; 160: 2765-72.

18. Kannel W, Wilson P, Nam B, D'Agostino R. A likely explanation for the J-Curve of blood pressure cardiovascular risk. Am J Cardiol 2004; 94: 380-4.

19. Franklin S, López V, Wong N, Mitchell G, Larson M, Vasan R, et al. Single versus combined blood pressure components and risk for cardiovascular disease. Circulation 2009; 119: 243-50.

20. Beddhu S, Chertow G, Cheung A, Cushman W, Rahman $\mathrm{M}$, Greene T, et al. Influence of Baseline Diastolic Blood Pressure on Effects of Intensive Compared With Standard Blood Pressure Control. Circulation 2018; 137: 134-43.

21. Messerli FH, Mancia G, Conti C, Hewkin A, Kupfer S, Champion A, et al. Dogma disputed: can aggressively lowering blood pressure in hypertensive patients with coronary artery disease be dangerous? Ann Intern Med 2006; 144: 884-93. 
22. Denardo S, Messerli F, Gaxiola E, Aranda J, Cooper-DeHoff R, Handberg M, et al. Coronary Revascularization Strategy and Outcomes According to Blood Pressure (from the International Verapamil SR-Trandolapril Study [INVEST]). Am J Cardiol 2010; 106: 498-503.

23. Bangalore S, Messerli F, Wun C, Zuckerman AL, DeMicco D, Kostis JB \& LaRosa JC. J-curve revisited: An analysis of blood pressure and cardiovascular events in the Treating to New Targets (TNT) Trial. Eur Heart J 2010; 23: 2897-908.

24. Hanson L, Zanchetti A, Carruthers S, Dalhöf B, Elmfeldt $\mathrm{D}$, Julius $\mathrm{S}$, et al. Effects of intensive blood-pressure lowering and low-dose aspirin in patients with hypertension: principal results of the Hypertension Optimal Treatment (HOT) randomised trial. Lancet 1998; 351: 1755-62.

25. Owens $\mathrm{P}, \mathrm{O}^{\prime}$ Brien E. Hypotension in patients with coronary disease: can profound hypotensives events cause myocardial ischaemic events? Heart 1999; 82: 477-81.

26. Vidal-Pierot E, Ford I, Greenlaw N, Ferrari R, Fox K, Tardif JC, et al. Cardiovascular event rates and mortality according to achieved systolic and diastolic blood pressure in patients with stable coronary artery disease: an international cohort study. Lancet 2016; 388: 2142-52.

27. UK Prospective Diabetes Study Group. Tight blood pressure control and risk of macrovascular and microvascular complications in type 2 diabetes: UKPDS 38. BMJ 1998; 317: 703-13.

28. Patel A, MacMahon S, Chalmers J, Neal B, Woodward M, Billot L, et al. Effects of a fixed combination of perindopril and indapamide on macrovascular and microvascular outcomes in patients with type 2 diabetes mellitus (the ADVANCE trial): a randomised controlled trial. Lancet 2007; 370: 829-40.

29. Cushman WC, Evans GW, Byington RP, Goff DC Jr, Grimm RH Jr, Cutler JA, et al. Effects of intensive blood-pressure control in type 2 diabetes mellitus. $\mathrm{N}$ Engl J Med 2010; 362: 1575-85.

30. ACCORDION: long-term follow-up of ACCORD patients. American Heart Association (AHA) 2015 Scientific Sessions, November 7-11, 2015; Orlando, Florida.

31. Redon J, Mancia G, Sleight P, Schumacher H, Gao P, Pogue J, et al. Safety and efficacy of low blood pressures among patients with diabetes: subgroup analyses from the ONTARGET (ONgoing Telmisartan Alone and in combination with Ramipril Global Endpoint Trial). J Am Coll Cardiol 2012; 59: 74-83.

32. Cooper-DeHoff R, Gong Y, Handberg E, Bavry A, Denardo S, Bakris G, et al. Tight Blood Pressure Control and Cardiovascular Outcomes Among Hypertensive Patients With Diabetes and Coronary Artery Disease.
JAMA 2010; 304: 61-8.

33. Estacio RO, Jeffers BW, Gifford N \& Schrier RW. Effect of blood pressure control on diabetic microvascular complications in patients with hypertension and type 2 diabetes. Diabetes Care 2000; 23 (suppl 2): B54-B64.

34. de Boer IH, Bangalore S, Benetos A, Davis AM, Michos ED, Muntner P, et al. Diabetes and Hypertension: A Position Statement by the American Diabetes Association. Diabetes Care 2017; 40: 1273-84.

35. Kidney Disease: Improving Global Outcomes (KDIGO) Blood Pressure Work Group. KDIGO Clinical Practice Guideline for the Management of Blood Pressure in Chronic Kidney Disease. Kidney Inter Suppl 2013; 3:1150.

36. Taler SJ, Agarwal R, Bakris GL, Flynn JT, Nilsson PM, Rahman M, et al. KDOQI US commentary on the 2012 KDIGO clinical practice guideline for management of blood pressure in CKD. Am J Kidney Dis 2013; 62: 20113.

37. Kovesdy CP, Bleyer AJ, Molnar MZ, Ma JZ, Sim JJ, Cushman WC, et al. Blood pressure and mortality in U.S. veterans with chronic kidney disease: a cohort study. Ann Intern Med 2013; 159: 233-42.

38. Palit S, Chonchol M, Cheung AK, Kaufman J, Smits G, Kendrick J. Association of BP with Death, Cardiovascular Events, and Progression to Chronic Dialysis in Patients with Advanced Kidney Disease. Clin J Am Soc Nephrol 2015; 10: 934-40.

39. Berl T, Hunsicker LG, Lewis JB, Pfeffer MA, Porush JG, Rouleau JL, et al. Impact of achieved blood pressure on cardiovascular outcomes in the Irbesartan Diabetic Nephropathy Trial. J Am Soc Nephrol 2005; 16: 2170-9.

40. Upadhyay A, Earley A, Haynes S \& Uhling K. Systematic review: blood pressure target in chronic kidney disease and proteinuria as an effect modifier. Ann Intern Med 2011; 154: 541-8.

41. Lazarus J, Bourgoignie J, Buckalew V, Greene T, Levey A, Milas N, et al. Achievement and safety of a low blood pressure goal in chronic renal disease. Hypertension 1997; 29: 641-50.

42. Norris K, Bourgoigne J, Gassman J, Hebert L, Middleton J, Phillips R, et al. AASK Study Group. Cardiovascular outcomes in the African American Study of Kidney Disease and Hypertension. Am J Kidney Dis 2006; 48: 739-51.

43. Ruggenenti P, Perna A, Loriga G, Ganeva M, Ene-Iordache B, Turturro M, et al. REIN-2 Study Group. Blood-pressure control for renoprotection in patients with non-diabetic chronic renal disease: multicenter, randomized controlled trial. Lancet 2005; 365: 939-46.

44. Peralta C, McClure L, Scherzer F, Odden M, White C, 
Shlipak M, et al. Effect of intensive versus usual blood pressure control on kidney function among individuals with prior lacunar stroke: a post hoc analysis of the Secondary Prevention of Small Subcortical Strokes (SPS3) randomized trial. Circulation 2016; 133: 584-91.

45. Cheung A, Rahman M, Reboussin D, Craven T, Greene $\mathrm{T}$, Kimmel P, et al. Effects of Intensive BP Control in CKD. J Am Soc Nephrol 2017; 28: 2812-23.

46. Malhotra R, Nguyen H, Benavente O, Mete M, Howard B, Mant J, et al. Association Between More Intensive vs Less Intensive Blood Pressure Lowering and Risk of Mortality in Chronic Kidney Disease Stages 3 to 5. A Systematic Review and Meta-analysis. JAMA Intern Med 2017; 177: 1498-505.

47. Bavry A, Anderson D, Gong Y, Denardo S, Cooper-DeHoff R, Handberg E, et al. Outcomes among hypertensives patients with concomitant peripheral and coronary artery disease: findings from the INternational VErapamil-SR/Trandolapil Study. Hypertension 2010; 55: 48-53.
48. Ovbiagele B, Diener HC, Yusuf S, Martin RH, Cotton D, Vinisko R, et al. Level of Systolic Blood Pressure Within the Normal Range and Risk of Recurrent Stroke. JAMA 2011; 306: 2137-44.

49. Juraschek S, Daya N, Appel L, Miller E, McEvoy J, Matsushita C, et al. Orthostatic Hypotension and Risk of Clinical and Subclinical Cardiovascular Disease in Middle-Aged Adults. J Am Heart Assoc 2018; 7:e00884, 15 May (10).

50. Poulter N, Castillo R, Charchar F, Schlaich M, Schutte A, Tomaszewski M, et al. Are the American Heart Association/Alerican College of Cardiology High Blood Pressure Guideliness Fit for Global Purpose?. Thoughts From the International Society of Hypertension. Hypertension 2018; 72: 260-2.

51. Williams B, Mancia G, Spiering W, Agabiti Rosei E, Azizi M, Burnier M, et al. 2018 ESC/ESH Guidelines for the management of arterial hypertension. European Heart J. 25 agosto 2018. https://doi.org/10.1093/eurheartj/ ehy339. 